\title{
Lecciones aprendidas en la provisión de medicamentos para la atención primaria de la salud
}

\author{
Federico Tobar, D en C Pol. ${ }^{(1)}$
}

\begin{abstract}
Tobar F.
Lecciones aprendidas en la provisión de medicamentos para la atención primaria de la salud. Salud Publica Mex 2008;50 supl 4:S463-S469.
\end{abstract}

\section{Resumen}

En las reformas de salud que se llevaron a cabo en América Latina, la provisión de medicamentos no adquirió protagonismo. Cuando fue incluida, se dio prioridad a los medicamentos para internación, así como para enfermedades de baja prevalencia y alto costo. Sin embargo, en los últimos años un conjunto de programas han realizado innovaciones en la gestión de suministros para el primer nivel de atención.Aquí se abordan estas experiencias en América Latina, primero analizándolas a través de cuatro momentos del ciclo de gestión de medicamentos: selección, compra, distribución y utilización. Después, se busca identificar las lecciones aprendidas y las condiciones de un buen sistema de provisión. Las conclusiones destacan experiencias que lograron no sólo disponibilidad a costos bajos, sino también aumentos en la productividad y resolutividad del primer nivel, además de mejoras en la prescripción y el fortalecimiento del funcionamiento en red.

Palabras clave: medicamentos para atención básica; acceso universal a servicios de salud; precio de medicamento, América Latina

\section{Tobar F.}

Lessons learned from drug supplies

for primary health care.

Salud Publica Mex 2008;50 suppl 4:S463-S469.

\begin{abstract}
Drug supply strategies have not been widely included in Latin American health reform programs. National efforts, when they have included such strategies, have focused on supporting drug availability for inpatient treatment and, in some cases, for ambulatory treatment of low prevalence and high-cost diseases. Nevertheless, some innovative drug supply programs for primary health care have been implemented in many countries. This article reviews and systematizes recent experiences with the supply of drugs in Latin American countries. Firstly, this article analyzes four phases of the cycle for managing drugs: selection, procurement, distribution, and use, and; secondly, it identifies lessons learned and some characteristics of efficient drug supply systems. Conclusions emphasize experiences which have not only achieved supply at low costs, but also have increased the productivity, efficiency, and effectiveness of primary health care, improved prescriptions, and strengthened the network.
\end{abstract}

Key words: drugs for primary health care; universal access to health care services; drug price; Latin America

(I) Centro de Estudios en Gestión y Economía de la Salud. Facultad de Ciencias Económicas, Universidad de Buenos Aires.Argentina.

Fecha de recibido: 9 de agosto de 2007 - Fecha de aprobado: I 4 de marzo de 2008 Solicitud de sobretiros: Dr. Federico Tobar. Centro de Estudios en Gestión y Economía de la Salud. Facultad de Ciencias Económicas, Universidad de Buenos Aires. Rodríguez Peña 608, Piso 10. Ciudad Autónoma de Buenos Aires (ADN I020),Argentina. Correo electrónico: federico@federicotovar.com.ar 
$\mathrm{E}_{\mathrm{p}}^{\mathrm{n}}$ América Latina se avanzó más en el acceso de las personas a los servicios médicos que a los medicamentos esenciales para el cuidado de su salud. La provisión pública y gratuita de medicamentos fundamentales a la población constituye una poderosa herramienta para promover el acceso y uso racional de los medicamentos. Aunque en todos los países de la Región el Estado despliega algún tipo de provisión, la incorporación de herramientas para su racionalización, así como para mejorar la eficiencia y la eficacia, resulta aún bastante limitada. Además, en la provisión de suministros se ha privilegiado al segundo y tercer nivel de atención con respecto al primero. Sin embargo, existen experiencias exitosas con aprendizajes que deben ser aprovechados.

En este artículo se identifican lecciones aprendidas a través de esas experiencias, y se parte de la distinción de cuatro momentos principales del ciclo de gestión de medicamentos: la selección, la compra, la distribución y la utilización. En cada uno se examinan las diversas experiencias nacionales y se concluye proponiendo respuestas a dos preguntas de investigación: ¿cuáles son las estrategias de provisión de medicamentos que mejor funcionan? y ¿cómo se sabe si las estrategias son adecuadas?

\section{La provisión de medicamentos en las agendas de políticas públicas}

En una evaluación de los logros obtenidos a través de las políticas de provisión pública, la Organización Mundial de la Salud concluyó que los resultados, entre 1978 y 1988, han sido muy dispares. Países tan distintos como Argelia, Costa Rica, Chipre, Indonesia, Kenia, Malasia, Papúa Nueva Guinea y Sri Lanka alcanzaron un éxito razonable en su selección, compra, distribución y utilización, mientras que en otros como Camerún, Costa de Marfil, El Salvador, Haití, Honduras, Madagascar, Nigeria y Túnez, los resultados han sido bastante deficientes. ${ }^{1}$

Es posible optimizar el rendimiento de las inversiones en sistemas de suministros para la provisión pública; esto implica obtener mejoras de eficiencia en las asignaciones, pero siempre dentro de un margen restricto. A través del análisis de experiencias internacionales, Management Sciences for Health concluyó que un programa de suministros típico, con un gasto anual del orden de un millón de dólares en medicamentos permite obtener, en promedio, un beneficio terapéutico para los pacientes equivalente a tan sólo 300 mil dólares estadounidenses. La falta de una selección cuidadosa, una cuantificación incorrecta, la adquisición a precios elevados, la deficiente calidad, los robos, un almacenamiento inadecuado, la caducidad de los productos, una prescripción irracional y una utilización incorrecta por parte de los pacientes, ocasionan pérdidas equivalentes a $70 \%$ de la inversión inicial. Según el mismo documento, con un esfuerzo considerable de gestión se pueden reducir dichas pérdidas a $40 \%$. Entre las mejoras que involucra la gestión de suministros se encuentran el desarrollo de sistemas de información y control y la adopción de mecanismos de almacenamiento adecuados y de guías (protocolos para promover la prescripción racional).

Aunque los beneficios son significativos, son todavía bastante limitados. No obstante, si se considera la gestión de suministros desde un enfoque más amplio de salud pública es posible obtener beneficios muy superiores. Se trata de lograr que la provisión de suministros no responda simplemente a la demanda orientada por los profesionales prescriptores, sino de consolidar un modelo de atención apropiado para el combate a las enfermedades prevalentes de una determinada población. En este sentido, el de suministros se constituye en un subsistema dentro de un esfuerzo social organizado para enfrentar dichas enfermedades prevalentes.

\section{Diseño de programas de provisión pública de medicamentos en América Latina}

La Organización Panamericana de la Salud promovió la traducción al castellano de un modelo para el análisis de la gestión de suministros, que distingue cuatro grandes momentos del "ciclo de gestión de medicamentos": selección, adquisición, distribución y uso. Se analizan a continuación las principales alternativas que, en cada momento, se plantean para el diseño de programas de provisión pública de medicamentos. ${ }^{2}$

\section{Selección racional}

Esta selección es un elemento del modelo de atención en salud que consiste en identificar los insumos necesarios para obtener la mejor respuesta frente a las necesidades de salud de la población. Casi todos los países de la Región disponen de un cuadro básico de medicamentos que se basa en la lista de medicamentos esenciales de la OMS. Sin embargo, en pocos casos la compra y la prescripción se ajustan a esas listas; en ocasiones existe más de una lista y muy pocas veces se han desagregado listas para cada nivel de atención. Esta última medida es una forma de forzar un modelo de división del trabajo en salud, así como los esquemas de referencia y contrarreferencia.

En la selección existen dos perspectivas clave: por un lado, la técnica que involucra capitalizar los conocimientos de medicina basada en la evidencia (MBE), llegando 
a definir algoritmos con guías, o al menos esquemas terapéuticos, que indiquen cómo se debe diagnosticar y tratar cada patología en cada nivel de atención. Por otro lado, la selección debe ser viable, esto es, debe ser aceptada por los actores involucrados en el proceso de atención médica y farmacéutica. De nada sirve contar con guías y medicamentos adecuados si los profesionales no los prescriben. Este problema se detectó en el Programa Remediar de Argentina, por ejemplo, con el sulfato ferroso y el ácido fólico, pues aunque la norma indica su prescripción a las embarazadas, los niveles de prescripción resultan ínfimos.

Para evitar este tipo de inconvenientes existen dos tipos de mecanismos: los preventivos y los correctivos. Ejemplo de los primeros es la convalidación, a través de eventos nacionales, del esquema terapéutico para cada problema de salud acompañado con una sólida capacitación en servicio sobre gestión clínica. Eso se puso en marcha en Paraguay con el Programa de Cuidados Sanitarios Básicos (PCSB) para 13 patologías prevalentes del binomio madre y niño. ${ }^{3}$ De igual manera, en República Dominicana se convalidaron, en un concurrido taller nacional, los esquemas terapéuticos para dar respuesta ambulatoria adecuada desde el primer nivel de atención a las necesidades de salud de la población beneficiaria del Plan Básico de Salud; a partir de dichos esquemas se seleccionaron 50 principios activos en 57 presentaciones.

Las medidas correctivas son mecanismos de auditorias médicas que buscan detectar errores de prescripción a través del análisis de recetas para luego implementar medidas de advertencia y sanción. En Costa Rica, la Caja Costarricense de Seguridad Social incorporó este análisis para alertar a los médicos, pero sin sanción. ${ }^{4} \mathrm{El}$ Programa Remediar de Argentina estableció un curso a distancia sobre prescripción racional, aunque aún no ha avanzado en la revisión de las recetas para retroalimentar dicha capacitación, ni ha evaluado su impacto sobre la mejora de las prescripciones.

En conclusión, la experiencia de la región demuestra que la MBE resulta insuficiente para concretar la selección racional, si ésta no va acompañada de medidas que garanticen la adhesión de los prescriptores y reduzcan la variabilidad de la práctica clínica. Por un lado, las medidas correctivas son aún muy incipientes y hay resistencia a ellas, pues a pesar de que se llegan a detectar desvíos, no se aplican sanciones a quienes no utilizan los medicamentos del listado o lo hacen en forma inadecuada. Mucho más provechosa ha resultado la experiencia con medidas preventivas como los eventos para validar listados y esquemas terapéuticos y, sobre todo, la capacitación en servicio a través de esquemas de supervisión capacitante. Este modelo fue incorporado por el PCSB en Paraguay en 2005 y luego en 2007 el ministerio decidió extenderlo a otros programas. A su vez, el Programa de Fortalecimiento de las APS (FEAPS) de Argentina se propuso incorporar esta supervisión capacitante a partir de 2008, para garantizar que las enfermedades cardiovasculares sean tratadas de forma homogénea en todas las redes locales. Por último, se comprueba que a mayor limitación en la lista de productos seleccionados, se obtienen mayores economías (ya que se requiere comprar menos productos), además de que se hace más viable la adhesión de los prescriptores a las listas y esquemas terapéuticos.

\section{Compra}

En la medida en que la demanda sea más agregada y la convocatoria más abierta, mejores resultan los precios obtenidos. En primer lugar, los precios menores corresponden a las compras realizadas para cubrir períodos extensos de operación, como un año, y realizadas a través de licitaciones públicas internacionales (LPI). En segundo lugar, las compras centralizadas a escala nacional permiten agregar demanda y facilitan la negociación de mejores precios para los productos. Sin embargo, durante toda la década de 1990 se promovió la descentralización de los servicios de salud en América Latina y, en consecuencia, se ha tendido a desagregar el poder de compra de los servicios. Incluso se destacaron las debilidades de los modelos de adquisiciones centralizadas, señalando que se corría el riesgo de comprar lo que no hacía falta a los servicios. En otras palabras, el argumento era que las compras centralizadas no favorecían la selección racional.

En la actualidad se han documentado diversas experiencias de adquisiciones centralizadas de insumos en América Latina por LPI que permitieron alcanzar economías significativas. ${ }^{5}$ Un ejemplo claro lo constituye el Programa Remediar de Argentina que obtiene precios hasta $90 \%$ inferiores a los minoristas vigentes en el mercado. ${ }^{6}$ En Paraguay, el PCSB provee medicamentos en los tres niveles de atención para cubrir de forma completa 13 patologías del binomio madre-niño. A través de la compra por LPI se obtuvieron ahorros del orden de $71.12 \%$ con respecto al precio de venta al público. ${ }^{7}$

Las compras a través de licitaciones nacionales presentan la ventaja de requerir plazos menores para su implementación, pero no detentan la misma capacidad de ahorro. En Perú, en 1994 se inició el Programa de Administración Compartida de Farmacias -a través del programa PACFARM- que adquirió medicamentos a precios entre 50 y $80 \%$ más bajos que los precios de los medicamentos originales vendidos en las farmacias. ${ }^{8}$ En Ecuador, el Seguro Social Campesino y el Ministerio 
de Salud Pública realizan licitaciones nacionales que permiten obtener los productos a valores inferiores en promedio al $40 \%$ de su precio de venta al público en farmacias minoristas. ${ }^{9}$

La eficiencia de las compras nacionales tiende a ser menor que la obtenida por LPI. Al evaluar los precios obtenidos en Ecuador, a través de compras nacionales en 2005, con los del programa argentino Remediar, en una compra internacional centralizada en el mismo año se verificó que con una LPI es posible obtener economías que oscilan entre 5 y $94 \%$ según el producto y, sobre el listado analizado, este mecanismo involucraría ahorros en una media del orden de 57\%. Dicho de otra manera, si en Ecuador se hubiera recurrido a una LPI que consiguiera precios similares a los obtenidos por el Programa Remediar se hubiera podido comprar una vez y media lo adquirido con los mismos recursos. ${ }^{9}$

Otra modalidad es la negociación conjunta en el plano nacional con compras descentralizadas. Durante 1996 este sistema permitió obtener descuentos de 74\% en Guatemala. ${ }^{10}$ El procedimiento de compra implementado en ese país ha sido denominado "contrato abierto" y comienza con la definición de las características del producto que, en todos los casos, evita la compra de marcas. El segundo paso consiste en establecer, de acuerdo con la demanda, cantidades orientadoras que deberán ser adquiridas en el ejercicio anual. El tercer paso se vincula con la convocatoria de propuestas de proveedores. Luego, una vez definidos los precios finales que ofrecen los proveedores (pueden presentarse hasta ocho proveedores para un mismo producto), cada unidad descentralizada define el volumen final de sus adquisiciones. Por otro lado, las compras centralizadas acompañadas de entregas descentralizadas de los insumos facilitan la construcción de un sólido sistema de información y control de gestión. Cuando la función de compra se descentraliza, es muy difícil consolidar información respecto a cuáles son las necesidades de insumos, cuáles son efectivamente adquiridos y provistos, y a qué precios. En Ecuador también se puso en marcha esta medida en 2005 y 2006 bajo el nombre de "Acuerdo de precios".

Dentro de las compras directas nacionales, una modalidad eficiente y bastante más ágil es la acreditación de proveedores y la convocatoria a una subasta invertida, que ha sido contemplada en Brasil (bajo el nombre de Leilão eletrônico), en Chile y en Colombia. Se ha estimado que los hospitales chilenos ahorraron aproximadamente cuatro millones de dólares estadounidenses utilizando este sistema, o sea de 5 a $7 \%$ de su presupuesto para medicamentos. ${ }^{11}$ En Colombia se establecieron consorcios de hospitales para aumentar las economías de escala en las adquisiciones. Además, el
Seguro Social consiguió descuentos promedio de 78.5\% en sus compras centralizadas durante 1999.*

Una mejor opción es agregar la demanda no sólo del sector público, sino también del privado y/o de los seguros sociales. Esto se ha concretado en Perú, donde la DIGEMID compra medicinas no sólo para el Ministerio deSalud, sino también para el Sistema Integrado de Salud (SIS). También ha sido contemplado en la creación de la Unidad Centralizada de Adquisiciones de Medicamentos y Afines de Estado (UCAMAE) del Uruguay buscando que adquiera para el Ministerio de Salud Pública y eventualmente, para las instituciones mutuales.

En conclusión, el aprendizaje que se desprende de esta experiencia es que resulta conveniente agregar compras -de preferencia en el plano nacional e incluyendo los servicios públicos y los de la seguridad social- y buscar la máxima competitividad en el proceso de adquisición, de ser posible a través de LPI.

\section{Distribución}

Se han identificado tres modelos alternativos en las experiencias recientes de la Región:

I. Integración de la adquisición y la distribución: en Ecuador, Guatemala y Panamá se ha establecido esta medida, mediante la cual el mismo proveedor se hace cargo de entregar los productos en los diferentes servicios del sistema de salud.

II. Distribución directa a cargo del financiador: la entidad que adquiere los medicamentos (ministerios o aseguradores) se hace cargo de su recepción en depósitos propios, los almacena y luego los distribuye a los servicios. Es la modalidad más frecuente en todos los ministerios de salud y también la que presenta mayores debilidades por pérdidas, vencimientos, robos y rotura de envases y productos. Esto también la vuelve poco ventajosa desde el punto de vista económico ya que, si bien se reducen las intermediaciones, también disminuye el aprovechamiento (que puede llegar a 50\% de los productos).

III. Operación logística por parte de un tercero: en este caso, el comprador delega la recepción, almacenamiento y distribución de los productos a un tercero u operador logístico. Este puede proveer la

\footnotetext{
* Arboleda R. La experiencia del Seguro Social de Colombia en la compra consolidada de medicamentos. En: Seminario Experiências Internacionais no controle dos preços dos medicamentos; 2000 mayo 8-10; Brasilia, Brasil. En prensa.
} 
reposición por insumo o por lotes (kits). La primera, que suele ser usada por las farmacias comerciales, resulta más cara debido a que exige la disponibilidad de un muy ágil sistema de información entre el centro de almacenamiento y procesamiento (operador logístico) y el servicio receptor de los insumos, además de que siempre implica una mayor cantidad de viajes entre el depósito y el servicio a ser provisto. A su vez, la distribución por lotes facilita la programación y organización de la distribución e incluso el cálculo de un costo unitario (por kit armado y entregado). Empero, introduce un riesgo mayor de que los servicios resulten desabastecidos o sobre inventariados.

En conclusión, una alternativa aconsejable es no limitarse a tercerizar la distribución para minimizar pérdidas y desvíos, sino también iniciar una estrategia gradual de procesamiento y distribución de los insumos por lotes estándar e ir configurando un sistema de información que permita optimizar la asignación de los insumos. De esta forma, se puede avanzar progresivamente hacia un esquema hecho a la medida.

\section{Utilización}

Únicamente en esta fase del ciclo de gestión del suministro, cuando se ha garantizado la disponibilidad, es posible generar un impacto sobre la calidad de vida de la población beneficiaria. La utilización involucra tres instancias: a) prescripción; b) dispensación y c) consumo.

a) Prescripción. Aunque en el mercado la receta es un requisito exclusivamente para una parte de los medicamentos, en los sistemas públicos de suministros se requiere que todas las medicinas entregadas a los pacientes generen y registren recetas correspondientes. Así, la receta se transforma en un documento muy valioso que permite verificar si la prescripción es adecuada a los protocolos normalizados o guías terapéuticas y si se realiza utilizando la Denominación Común Internacional (DCI); por otra parte, también provee información epidemiológica sobre incidencia y prevalencia de enfermedades. Una innovación ha consistido en estructurar la prescripción a partir de formularios de receta estandarizados que enumeran el listado de medicamentos para ese nivel de atención -de modo que el médico sólo debe marcar con una cruz el producto indicado- y que obligan a completar otros datos del paciente. Esto se implementó en Remediar y en el PCSB y se propuso en República
Dominicana para la Asistencia Farmacéutica Básica (AFB), pero luego fue rechazado por el Consejo Nacional de Seguridad Social.

b) Dispensación. Más que la simple entrega de un producto, la dispensación forma parte de la noción de atención farmacéutica que involucra la participación activa del farmacéutico en la orientación y seguimiento de un tratamiento farmacoterapéutico, cooperando así con el médico y otros profesionales sanitarios a fin de conseguir resultados que mejoren la calidad de vida del paciente. Las buenas prácticas de dispensación incluyen la recepción y almacenamiento adecuado de los suministros, la entrega oportuna al beneficiario, el control de inventarios y el llenado de los formularios elaborados para el monitoreo del sistema y, en especial, la orientación al usuario sobre los riesgos y los beneficios que involucra el tratamiento, así como sobre recaudos en su seguimiento. El Programa Remediar y el PCSB optaron por dispensar directamente en los servicios de salud: en la Ciudad de Sao Paulo, Brasil, se crearon dispensarios en diversos puntos de la ciudad, incluyendo las estaciones de trenes subterráneos. Pero el diseño más complejo se asumió en el programa dominicano de Asistencia Farmacéutica Básica (AFB) que incorporó una red de 500 Farmacias del Pueblo con responsabilidad nominada sobre la población a cargo, a quienes deben distribuir gratuitamente los medicamentos.

c) Consumo. Se utiliza este término para hacer referencia a la utilización del medicamento (y eventualmente del insumo no medicamentoso) por parte del paciente. Cuando el análisis de los medicamentos se centra en el paciente, los principales problemas son el acceso y la automedicación. Sin embargo, los sistemas de suministro público y gratuito resuelven ambos, ya que están diseñados para garantizar el acceso y únicamente permiten la distribución como resultado de una prescripción profesional. Por lo tanto, el tema más relevante en este caso es la cuestión del compliance o adherencia al tratamiento indicado. En este sentido, la tarea del prescriptor y del dispensador de sensibilizar al paciente es fundamental. La inclusión de prospectos médicos en los envases secundarios de los productos también constituye una medida de seguridad adicional. Se logra tener más cuidado en el consumo mediante acciones de comunicación masiva dirigidas a los pacientes (información, educación y comunicaciónIEC). En los Estados Unidos de América la farmacopea ha desarrollado folletos para los pacientes con información específica acerca de la enfermedad diagnosticada y sus posibles tratamientos, redac- 
tados en un lenguaje accesible al público lego. Por último, una medida extrema de control consiste en auditar los hogares de los beneficiarios para verificar junto a ellos la adherencia al tratamiento y el uso correcto de los productos.

\section{Lecciones aprendidas}

Se pueden sintetizar las lecciones aprendidas con la revisión de las experiencias de provisión pública de medicamentos en América Latina, respondiendo a dos interrogantes que a continuación se abordan.

1) ¿Cuáles son las estrategias de provisión de medicamentos que mejor funcionan?

Todas aquellas que consiguen universalizar el acceso a bienes y servicios de salud a toda la población y, al mismo tiempo, contener los costos y mejorar la eficiencia en la asignación de recursos. Las experiencias más innovadoras en América Latina han sido aquellas donde se concibió la asistencia farmacéutica como una oportunidad para reformular el modelo de atención. Esto significa mucho más que proveer suministros, ya que a través de los medicamentos se han logrado objetivos como aumentar la productividad y resolutividad de los servicios del primer nivel, mejorar la prescripción, reducir la incidencia de cuadros que requieren atención especializada e internación y mejorar el sistema de referencia y contrarreferencia.

Esta articulación se concreta en las cuatro fases del ciclo de gestión del medicamento. En la selección, porque es el momento de concentrar esfuerzos en un conjunto limitado de patologías y medicamentos que posibiliten la prevención y el tratamiento adecuado en cada nivel de atención. En la compra, porque es la instancia que debe garantizar la economía de escala y la calidad del producto. En la distribución, porque ahí es donde se debe garantizar la equidad, de modo que todos los servicios dispongan en tiempo y forma de los medicamentos adecuados a su nivel de atención. En la utilización, porque es la etapa donde se concreta la acción terapéutica y sin los recaudos necesarios quedaría trunca.

\section{2) ¿Cómo saber si las estrategias son adecuadas?}

En función de la revisión de las experiencias recientes en la Región, se puede afirmar que una estrategia de provisión de medicamentos para atención primaria de la salud (APS) es adecuada cuando pone a disposición insumos de acuerdo con perfiles asistenciales bien definidos para cada nivel de atención, y garantiza su provisión gratuita a la población. De esta manera, se organiza la oferta y se orienta la demanda hacia un sistema de cuidados progresivos donde cada servicio no hace de todo sino, más bien, sólo lo que tiene que hacer. En conclusión, se pueden enumerar siete condiciones que un buen sistema de provisión de suministros para APS debe cumplir:

1. Generar beneficios significativos en términos de productividad y resolutividad de los servicios. Por ejemplo, a través de la provisión de kits con medicamentos a los Centros de APS en Argentina, el Programa Remediar consiguió incrementar, únicamente en su primer año (2003) 50\% del volumen de consultas de estos centros. Incrementos similares se registraron en República Dominicana entre 2004 y 2005 con el Fondo para fortalecimiento de la APS (FONAPS) que, con un préstamo del BID, financió servicios de primer nivel integrados por prestadores públicos y no gubernamentales en tres provincias.

2. Fortalecer el funcionamiento en red. Esto se logra en la medida en que un medicamento de APS no se encuentre disponible en servicios de mayor complejidad y viceversa. Al evitar que los servicios de diferentes niveles compitan entre sí por los suministros, es más fácil lograr que cada uno haga lo que tiene que hacer. Por ejemplo, el Programa de Cuidados Sanitarios Básicos de Paraguay provee periódicamente cinco tipos de kits con suministros a los servicios: kits para puestos, para centros, para hospitales, de parto normal y de cesárea. En la medida que los componentes de cada uno son diferentes, se logra consolidar un modelo de atención que divide el trabajo de forma coherente en el combate a las principales causas de muerte materna e infantil.

3. Fortalecer acciones preventivas y de tratamiento precoz. Aquí cabe citar a la fundación Tzedaká de Argentina que, al evaluar el impacto de su Banco Comunitario de Medicamentos, concluyó que por cada dólar invertido en garantizar la provisión continua de medicamentos para el tratamiento de la hipertensión arterial, obtiene economías del orden de casi 17 dólares evitados en la atención de infartos agudos de miocardio y accidentes cerebrovasculares. ${ }^{12}$ Por su parte, el Programa de Cuidados Sanitarios Básicos de Paraguay se tradujo en un impacto concreto sobre la salud de sus beneficiarios. Por ejemplo, los días que las madres de niños menores de cinco debieron ausentarse de su trabajo porque sus hijos sufrían infecciones respiratorias agudas se redujeron de un promedio 
de 8.1 antes del programa a 3.4 al año de su implementación. ${ }^{13}$

4. Generar informaciones estratégicas. La incorporación de un sistema de información capaz de monitorear las prescripciones con sus correspondientes diagnósticos permite generar herramientas no sólo para el control de los insumos sino también para la vigilancia sanitaria y epidemiológica. Esto facilita la toma de decisiones para realizar eventuales ajustes acerca de las prestaciones que es necesario reforzar y en qué servicios.

5. Promover la calidad de la atención. Al concentrar la provisión en un elenco restricto y consensuado de suministros, es posible capacitar y sensibilizar a los profesionales para promover una mejor prescripción e, incluso, controlar la utilización y el seguimiento de los tratamientos por parte de los pacientes.

6. Reducir los costos de la atención médica. Todas las anteriores razones permiten estimar que las economías que pueden ser generadas al articular la gestión de suministros con la implementación de un modelo de atención adecuado potenciarían la inversión inicial, duplicando el beneficio de la gestión de suministros e incrementando su rendimiento.

7. Mejorar la equidad social. Otro argumento económico positivo es que la provisión gratuita de medicamentos para el primer nivel de atención registra un altísimo impacto redistributivo. Esto se debe a que el financiamiento de los medicamentos depende considerablemente de los ingresos de los hogares y ésta es una forma de financiamiento muy regresiva, ya que los sectores de menores ingresos, que en general son los que registran mayores necesidades, destinan proporciones significativas de sus ingresos para adquirir medicamentos. En América Latina y el Caribe, en general, el gasto privado en salud es proporcionalmente muy alto. ${ }^{14}$ En Argentina se ha evaluado el impacto del Programa Remediar sobre el financiamiento de medicamentos y se concluyó que, a partir de la puesta en marcha de este esquema de provisión pública, el gasto en medicamentos resulta distribuido de forma mucho más equitativa entre los diferentes quintiles de ingreso ${ }^{15}$ y se refleja en una reducción de $60 \%$ del coeficiente de Gini, que mide la concentración de dicho gasto.

\section{Referencias}

I.World Health Organization. The world drug situation. Geneva: WHO, 1988.

2. Management Sciences for Health. La gestión de suministros de medicamentos: selección, compra, distribución y utilización de productos farmacéuticos. 2a. edición. Boston: Management Sciences for Health, 2002. 3. Ministerio de Salud Pública y Bienestar Social de la República de Paraguay. Manual de procedimientos. Programa de Cuidados Sanitarios Básicos. Asunción: Ministerio de Salud Pública y Bienestar Social de la República de Paraguay, 2006.

4. Chaves A. Estudios de utilización de medicamentos: aplicaciones. En: Zepeda-Bermúdez J,Alacantara-Bonfim J, eds. Medicamentos e a reforma do setor saúde. San Pablo: Hucitec-Sobravime, 1999: 115.

5. Tobar F. Políticas de provisión pública de medicamentos en América Latina y el Caribe. Med Soc 2007;6:2. Disponible en: http://www.medicinaysociedad.org.ar/publicaciones/25_junio2007/25junio.htm

6. Tobar F. Políticas para promoción del acceso a medicamentos: el caso del Programa Remediar de Argentina. Nota técnica de discusión de salud 002/2004. Washington DC: Banco Interamericano de Desarrollo, 2004.

7. Ministerio de Salud Pública y Bienestar Social de la República de Paraguay. Comparativo de precios de venta al público y costo de medicamentos de la Licitación Pública Internacional 03/2005. Programa de Cuidados Sanitarios Básicos. Asunción: Ministerio de Salud Pública y Bienestar Social de la República de Paraguay, 2006.

8. Phang-Romero $C$. Reforma del sector salud y la política farmacéutica en Perú. Cad Saude Publica 2002; I8 (4): I I I - I I 38.

9. Representación en Ecuador del Banco Interamericano de Desarrollo. Análisis y propuestas de reforma del sistema de medicamentos e insumos médicos en el Ecuador. Quito: Representación en Ecuador del Banco Interamericano de Desarrollo, 2006: 63-67.

10. Lezama-Minera M. Memoria de la transformación del proceso de atención, disponibilidad y acceso a medicamentos y otros insumos. Guatemala: Ministerio de Salud Pública y Asistencia Social de la República de Guatemala, 1999.

II. Cohen J, Carikeo-Montoya J. Using technology to fight corruption in pharmaceutical purchasing: lessons learned from the Chilean experience Washington DC: The World Bank, 200 I.

12. Fundación Tzedaka. Evaluación de Banco Comunitario de Medicamentos Refuot. Buenos Aires: Fundación Tzedaka, 2006. 13. Ministerio de Salud Pública y Bienestar Social de la República de Paraguay. Evaluación: Encuesta domiciliaria de utilización y gasto con medicamentos y servicios de salud. Paraguay: Bienestar Social de la República de Paraguay, 2007.

14. Inter-American Development Bank. Sustaining development for all: expanding access to economic activiy and social services. Washington DC: Inter-American Development Bank, 2006: I57-I58.

I5. Falbo R. Impacto redistributivo del Programa Remediar. En: Comisión Nacional de Programas de Investigación Sanitaria, comp. El futuro del acceso a los medicamentos en Argentina. Buenos Aires: Comisión Nacional de Programas de Investigación Sanitaria del Ministerio de Salud de la Nación, 2004: 77-96. 